\title{
Sistema de Despacho Aduanero y su importancia en la Cadena Logística del Comercio Internacional
}

\author{
Customs Clearance System and their Importance \\ in the International Trade Supply Chain
}

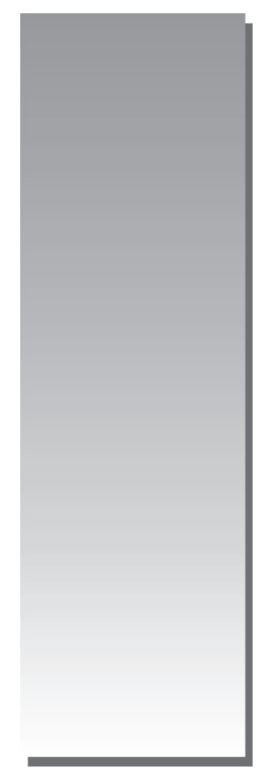

Mg. Ricardo Aspilcueta A.* raasolimana@hotmail.com Lic. Adm. NN.II. José Bazán T.**** jabaztan@hotmail.com

[RECEPCIÓN: AGOSTO 2016 / CONFORMIDAD: OCTUBRE 2016]

\section{RESUMEN}

La investigación tiene como objetivo analizar la normativa y los procedimientos de despacho aduanero implementados por SUNAT y cómo influye en la dinámica de los distintos operadores de comercio exterior; identificando en primer lugar a los operadores que participan en la cadena logística, los regímenes aduaneros, las modalidades de despacho aduanero, para finalmente identificar aquellas operaciones aduaneras que, de alguna manera, traban la facilitación al comercio internacional, los cuales tendrían un impacto negativo en las operaciones aduaneras en la cadena logística del comercio exterior. La investigación alcanzó un nivel descriptivo y correlacional, basada en la información obtenida de SUNAT y de la literatura especializada. Como resultado de la investigación, se logró determinar que es necesario llevar a cabo la adecuación de ciertas actividades operativas y aduaneras de la cadena logística de comercio exterior, ya que nuestro país se encuentra en un franco proceso de crecimiento y dinamización de esta actividad.

Palabras clave: despacho aduanero, operadores de comercio exterior, cadena logística, regímenes aduaneros, operaciones portuarias.

\section{ABSTRACT}

The research aims to analyze the regulations and procedures of customs clearance implemented by SUNAT and how it influences the dynamics of the different foreign trade operatos; firstly identifying the operators involved in the logistics chain, custom regimes, customs clearance modalities, to finally identify those customs operations that in some way obstruct the facilitation of international trade, which would have a negative impact on the operations Customs in the foreign trade logistics chain. The research reached a descriptive and correlational level, based on the information obtained from SUNAT and the specialized literature. As a result of the investigation it was possible to determine that it is necessary to carry out the adaptation of certain operational and customs activities of the

\footnotetext{
* Ingeniero industrial. Magíster en Gestión Turística Territorial. Doctorando en Ciencias Administrativas. Diplomado en Comercio Exterior por ADEX. Docente universitario en la Facultad de Ciencias Administrativas - UNMSM.

** Licenciado en Administración de Negocios Internacionales, Facultad de Ciencias Administrativas - UNMSM. Maestría en Ciencias de la Gestión Económica Empresarial, Facultad de Ciencias Económicas - UNMSM. Master Management por la Université de Bordeaux - Francia. Docente del curso de Comercio Internacional e Introducción a la Administración, Finanzas y Negocios Globales en U. P. TELESUP.
} 
foreign trade logistics chain since our country is in a frank process of growth and dynamization of this activity.

Keywords: customs clearance, foreign trade operators, supply chain, customs procedures, port operations.

\section{INTRODUCCIÓN}

En la actualidad, existe en nuestro país una clara conciencia de la importancia del comercio exterior por el creciente interés que se le está dedicando, tanto por los organismos gubernamentales como intergubernamentales. Tal vez, su importancia recién se ha hecho notar como una presencia dinámica dentro del sector comercio, en respuesta al crecimiento que existe en las inversiones de mayor dimensión en nuestro país, cuya fundamental tarea es exportar para atender la demanda internacional e importar para atender la demanda nacional.

En los países emergentes como el nuestro, y debido a la falta de recursos financieros para la inversión productiva en escalas considerables, SUNAT se presenta como la única vía posible para implementar un ágil comercio internacional, pero no solo como una forma inevitable, sino como un medio racional y eficaz, a través del cual pueden ser convenientemente orientadas las iniciativas empresariales privadas.

Esta realidad nos ha motivado a plantear esta investigación, cuyo objetivo principal es analizar si la administración aduanera, a través del perfeccionamiento de su normativa y procedimientos, influye directamente en los distintos operadores de comercio exterior al hacer que estos puedan ofrecer y prestar servicios más complejos de una forma más ágil y dinámica, y, de esta forma, impactar de manera positiva en las operaciones aduaneras en la cadena logística del comercio exterior.

\section{ANTECEDENTES}

Antes de analizar el impacto en la cadena logística que tiene el sistema actual de despacho aduanero, resulta necesario hacer una reseña histórica de la Aduana, la cual está estrechamente ligada a la historia portuaria de nuestro país. Así, en el año 1773 se promulgó el primer Reglamento de Comercio y Aduanas, otorgándole el debido ordenamiento legal a las funciones aduaneras (Huamán, 2011). Con el transcurso de los años se creó ADUANAS, a través del Decreto Ley $\mathrm{N}^{\circ} 26020$
(Ley de Creación de la Superintendencia Nacional de Aduanas, 1992), organismo responsable de, entre otras funciones:

- Facilitar las actividades de comercio exterior.

- Cautelar el cobro real y justo de los tributos que gravan las operaciones aduaneras.

- Fiscalizar el tráfico internacional de mercancías, medios de transporte y personas.

- Proporcionar estadísticas confiables a los operadores de comercio exterior.

Bajo este contexto, las normas aduaneras siempre han puesto énfasis al ingreso y la salida de mercancías y a los distintos regímenes y operaciones aduaneras vinculadas a este flujo. Para el caso de los procedimientos aduaneros de importación, antes de la promulgación de la Ley $\mathrm{N}^{\circ} 24829$ (Ley de Creación de la Superintendencia Nacional de Aduanas, 1988), las aduanas tenían el monopolio de las funciones propias de los servicios portuarios. Tomando en cuenta las funciones que se ejercían por las aduanas, el control fiscal necesariamente se hacía en los almacenes del propio puerto y eran las vistas de aduanas las únicas autoridades competentes para llevar a cabo dichas diligencias. De forma regular, el aforo de las mercancías siempre se hacía en el propio puerto, con excepción de las mercancías que por su naturaleza requerían un tratamiento especial e inmediato, circunstancias que resultaban óptimas, dado que las mercancías permanecían dentro del puerto, llevándose a cabo todas las diligencias aduaneras en el mismo lugar, evitándose que estas sean enviadas a zonas extra-portuarias.

Este sistema operativo para el aforo de las mercancías en el mismo puerto se mantuvo por muchos años, no obstante, a partir del año 1970 , la Aduana deja la función de la recepción de las mercancías, así como del almacenamiento y custodia, dado que estas le fueron delegadas a la Empresa Nacional de Puertos (ENAPU), en el caso de transporte marítimo. Sin embargo, la falta de prevención por parte de ENAPU, al no preparar- 
se para la etapa de la contenedorización, es lo que produjo un cambio de sistema, dado que los puertos de nuestro país no contaban con la logística necesaria para efectuar operaciones con contenedores, sumándose a dicha falta, la de zonas especialmente preparadas para su almacenamiento. Esta circunstancia obligó a dicha entidad a adaptar una pequeña zona de contenedores que, ciertamente, resultaba insuficiente, a tal punto que se tuvo que recurrir al apoyo de las empresas privadas para poder atender el movimiento de los contenedores en continuo ascenso. Ante este panorama, el Gobierno peruano autorizó la creación de lo que se conoce como extensiones del puerto, las cuales se denominaron terminales de almacenamiento.

Las medidas adoptadas cambiaron sustancialmente el sistema de las operaciones portuarias, porque la carga tenía que salir del puerto para ser trasladada a estos terminales de almacenamiento, apareciendo así el riesgo de los robos o pérdidas a que estaban expuestas las mercancías durante el traslado hasta llegar al terminal de almacenamiento "extraportuario".

En los años noventa, la Aduana desarrolló un vasto programa de reforma institucional. Como parte de dicho proceso, se buscó la reducción de los tiempos de desaduanaje de las mercancías, implementando el procedimiento denominado Sistema Anticipado Preferente (SAP), creado para empresas con altos volúmenes de operación, a fin de que puedan desaduanar su mercancía en el mismo día de llegada, permitiendo una sustantiva disminución de costos operativos.

No obstante, en el año 2002, se produjo la fusión por absorción entre la entonces Superintendencia Nacional de Aduanas con la Superintendencia Nacional de Administración Tributaria (Decreto Supremo $\mathrm{N}^{\circ}$ 061-2002-PCM), bajo el fundamento de maximizar las labores de recaudación y fiscalización de los contribuyentes y ahorrar importantes recursos al Estado para financiar el desarrollo de estas actividades que estaban siendo asumidas por ambas entidades hasta ese momento.

En el año 2004, se iniciaron las negociaciones con Estados Unidos para la suscripción del Tratado de Libre Comercio, lo que conllevó replantear y adecuar una serie de mecanismos de cara a la implementación de este acuerdo. Entre ellos se encuentran, la normativa aduanera con el fin de garantizar la facilitación del comercio exterior, promulgándose diversas normas tales como la Ley $N^{\circ} 28977$ (Ley de Facilitación del Comercio Exterior, 2004) hasta el vigente Decreto Legislativo $\mathrm{N}^{\circ} 1053$ (Ley General de Aduanas, 2008), y sus normas y procedimientos afines.

\section{MÉTODOS}

La presente investigación se abordó bajo un enfoque cualitativo, como un estudio documental compuesto por dos etapas: una primera etapa descriptiva y otra explicativa. En la primera etapa se llevó a cabo el examen de la información cuantitativa y cualitativa existente sobre los factores que inciden en la dinámica del comercio exterior peruano y que generan la necesidad de acelerar las operaciones portuarias, así como la de simplificar las actividades aduaneras. La etapa explicativa se formó a través de la revisión documental y procedimental como técnica para profundizar en la discusión dirigida a construir explicaciones para analizar el rol que tiene el actual sistema de despacho aduanero y cómo incide en la cadena logística del comercio internacional.

\section{RESULTADOS}

\section{Operadores de comercio exterior}

Los operadores que participan en las operaciones de comercio exterior y que se encuentran regulados bajo la normativa aduanera son:

Despachadores de aduanas; los transportistas o sus representantes en el país; los agentes de carga internacional; almacenes aduaneros; Empresas de servicio postal; empresas de servicio de entrega rápida; almacenes libres (Duty Free); beneficiario de material de uso aeronáutico y usuario aduanero certificado.

\section{Regímenes aduaneros}

La Ley General de Aduanas vigente contempla los siguientes regímenes aduaneros:

Regímenes de importación, regímenes de exportación, regímenes de perfeccionamiento, régimen de depósito y régimen de tránsito.

Para efectos del presente estudio, nos enfocaremos en los regímenes de importación para el consumo y la exportación definitiva, puesto que son los principales regímenes utilizados en las operaciones de comercio exterior; no obstante, se debe mencionar que los otros regímenes varían 
según lo solicitado por el importador/exportador, atendiendo a sus necesidades, destino, entre otros.

\section{Exportación definitiva}

Este régimen aduanero permite la salida del territorio aduanero de las mercancías nacionales o nacionalizadas para su uso o consumo definitivo en el exterior, el cual no está afecto a ningún tributo.

En la exportación definitiva, las mercancías deben ser embarcadas dentro del plazo de treinta (30) días calendario, contados a partir del día siguiente de la numeración de la declaración aduanera de mercancías. La regularización del régimen se realizará dentro del plazo de treinta (30) días calendarios contados a partir del día siguiente de la fecha del término de embarque.

De acuerdo a la Ley General de Aduanas y el Procedimiento General de Exportación Definitiva (INTA.PG.02), toda mercancía a embarcarse con destino al exterior debe ser puesta bajo potestad aduanera, para lo cual debe ingresar a un depósito temporal portuario o extraportuario, excepto aquellas mercancías tales como la carga peligrosa, carga de gran volumen, carga a granel, animales vivos, que se encuentran señalados en el procedimiento citado.

De acuerdo a la información obtenida en otros estudios (MINCETUR, 2005), se ha determinado que el costo por el servicio de depósito temporal que brindan los propios terminales portuarios es menor que los cobrados por los depósitos temporales extraportuarios.

Sin embargo, lo cierto es que a pesar de la diferencia existente entre los costos del depósito temporal portuario y el extraportuario, la mayor cuota de mercado por este servicio la tienen los depósitos temporales extraportuarios, conforme se puede apreciar en el siguiente gráfico $\mathrm{N}^{\circ} 01$ :

\section{Gráfico $\mathrm{N}^{\mathrm{O}} 01$}

Cuotas de mercado en el servicio de depósito Temporal de contenedores llenos de exportación por terminal de almacenamiento, 2010 - 2013.

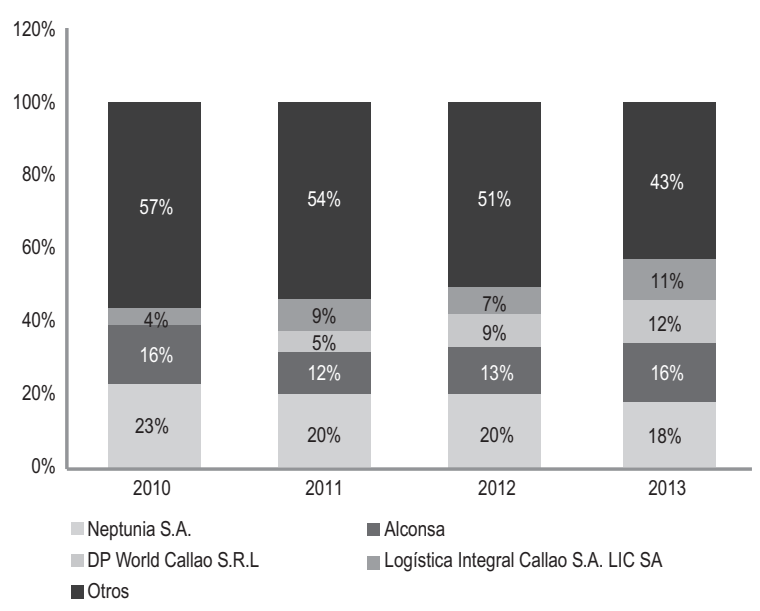

Fuente: Memorias Anuales SUNAT

Elaboración: Apoyo Consultoría

\section{Importación para el consumo}

Este régimen aduanero permite el ingreso de mercancías al territorio aduanero, luego del pago o garantía -según corresponda- de los derechos arancelarios y demás impuestos aplicables, así como el pago de los recargos y multas que hubiera, y del cumplimiento de las formalidades y otras obligaciones aduaneras (INTA.PG.01., 2009). Las mercancías extranjeras se considerarán nacionalizadas cuando haya sido concedido el levante.

\section{Modalidades de despacho aduanero}

Las mercancías que ingresan a nuestro país serán sometidas a los regímenes aduaneros cuando los despachadores de aduana u otras personas autorizadas legalmente soliciten la destinación aduanera dentro del plazo de quince (15) días calendario antes de la llegada del medio de transporte. Sin embargo, excepcionalmente se podrá solicitar la destinación aduanera hasta los treinta 
(30) días calendarios posteriores a la fecha del término de la descarga. Transcurrido dicho plazo sin haber solicitado la destinación aduanera, se producirá el abandono legal a favor del Estado.

Cabe precisar que la destinación aduanera se realiza a través de la Declaración Aduanera de Mercancías (DAM), la cual es tramitada de acuerdo a las necesidades propias de cada consignatario o dueño de la carga, bajo las modalidades de despacho comprendidas en la Ley General de Aduanas, que a continuación se detalla:

- Despacho anticipado

- Despacho urgente

- Despacho excepcional (Diferido)

\section{a. Despacho anticipado}

En el despacho anticipado, las mercancías pueden ser solicitadas al régimen de importación dentro del plazo de quince (15) días calendario antes de la llegada del medio de transporte, pudiendo disponer de la mercancía desde el mismo puerto o su traslado inmediato al almacén del dueño o consignatario para las acciones de control respectivas, sin la necesidad de ingresar a un depósito temporal.

\section{b. Despacho urgente}

En el despacho urgente, a diferencia del despacho anticipado, las mercancías pueden ser solicitadas al régimen de importación para el consumo dentro del plazo de quince (15) días calendario antes de la llegada del medio de transporte hasta los siete (07) días calendario computados a partir del día siguiente del término de la descarga; asimismo, por su naturaleza, no pueden permanecer en el puerto o terminal, debiendo ser retiradas inmediatamente.

\section{c. Despacho excepcional (Diferido)}

En el despacho excepcional, las mercancías pueden ser solicitadas al régimen de importación para el consumo dentro del plazo de treinta (30) días calendario computados a partir del día siguiente del término de la descarga. De exceder dicho plazo, las mercancías caerán en abandono legal.
Si bien la diferencia entre estas modalidades de despacho radica en la oportunidad en la que la mercancía por arribar o arribada es sometida al régimen de importación, ello también implica una diferencia en los costos operativos en que incurre un importador, puesto que no solo le permite disponer de su mercancía en el menor tiempo posible, sino que, además, reduce algunos costos como el almacenamiento, transporte, entre otros. Se puede afirmar, entonces, que la utilización del despacho anticipado beneficia a los importadores, en cuanto existe una considerable reducción de determinados costos, sin embargo, existen algunos factores que impiden y hacen poco ágil y, consecuentemente, poco atractiva su utilización. Previamente a la identificación de estos factores, se hará el relato sobre el desarrollo que ha sufrido el sistema de despacho aduanero en nuestro país.

\section{Evolución del sistema de despacho aduanero y sus limitaciones}

\section{a. Despacho anticipado}

Como ya se definió anteriormente, mediante la modalidad de despacho anticipado se permite que el despachador de aduanas pueda numerar la declaración aduanera y destinar la mercancía antes del arribo de la nave. Si bien esta modalidad no es nueva, pues, como se señaló en los antecedentes, se trató de una de las reformas que se plantearon en los años noventa con el Sistema Anticipado Preferente (SAP) y luego con el Sistema Anticipado de Despacho Aduanero (SADA), estos sistemas se concibieron como modalidades que eran usadas solo de manera excepcional, pues al permitir que el importador dispusiera de su mercancía inmediatamente, e incluso llevarla a su propio almacén para las acciones de control (aforo físico). Los requisitos para acceder a este sistema estaban restringidos básicamente a aquellos importadores que ingresaran grandes volúmenes de carga, haciendo difícil, de esta manera, su acceso al resto de importadores.

Sin embargo, como se puede observar en los gráficos $\mathrm{N}^{\circ} 02, \mathrm{~N}^{\circ} 03$ y 04, en los años 2005 al 2007, la utilización del SADA era mínima, viéndose un incremento recién a partir del año 2008. 


\section{Gráfico N ${ }^{\circ} 02$}

DAM numeradas por modalidad de despacho anticipado, $2005-2010$.



Fuente: VeritradeAnalytics

Elaboración: Apoyo Consultoría

\section{Gráfico N ${ }^{\circ} 03$}

Evolución del despacho anticipado, 2005 - 2010.

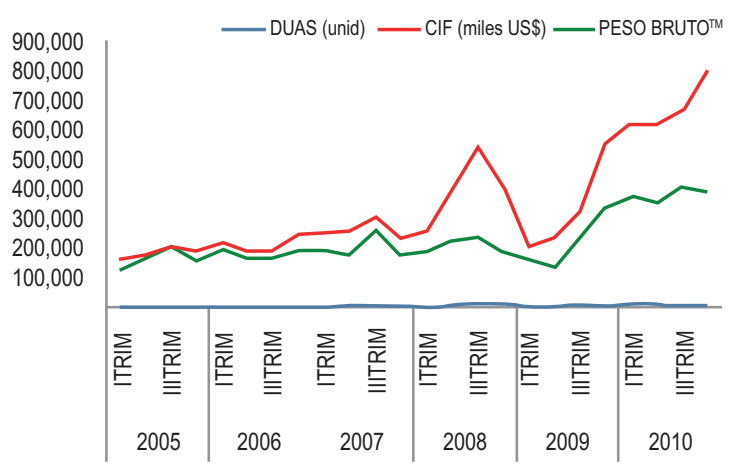

Fuente:VeritradeAnalytics

Elaboración: Apoyo Consultoría

Ahora bien, con la promulgación del Decreto Legislativo $N^{\circ} 1053$ (Ley General de Aduanas, 2008) y de cara a las negociaciones del Tratado de Libre Comercio con Estados Unidos y otros países, se concibió el concepto del levante dentro de las cuarenta y ocho (48) horas. Así, por ejemplo, en el artículo 167 de la actual Ley General de Aduanas se establece que "la autoridad aduanera dispondrá las acciones necesarias para que, en la medida de lo posible, las mercancías puedan ser de libre disposición dentro de las cuarenta y ocho (48) horas siguientes al término de la descarga".

Para lograr esto, el despachador de aduanas, el transportista, el agente de carga internacional y el dueño o consignatario deben, cada uno desde su obligación aduanera, cumplir los siguientes requisitos:

- Contar con garantía global o específica previa a la numeración de la declaración.

- Transmitir el manifiesto de carga antes de la llegada del medio de transporte.

- Numerar la declaración antes de la llegada del medio de transporte.

- Contar con toda la documentación requerida por la legislación aduanera para el despacho de las mercancías, tales como permisos, facturas, entre otros.

- No se haya dispuesto sobre la mercancía una medida preventiva de inmovilización o incautación, o la suspensión del despacho por aplicación de medidas en frontera.

- Transmitir la nota de tarjeta hasta ocho (8) horas siguientes al término de su descarga.

La modalidad de despacho anticipado permite que el importador pueda retirar su mercancía desde el punto de llegada, o ser trasladada a su propio almacén, en donde se realizarán las acciones de control respectivas. Ahora, de información obtenida por SUNAT, se ha podido observar que, en términos de tiempo de despacho, en el $80 \%$ de las declaraciones anticipadas se otorga el levante antes de que llegue la carga o el mismo día del arribo, siendo el tiempo de demora no superior a un día. Así, tenemos también que otro $16.14 \%$ son canal naranja, cuyo levante se otorga desde antes que la carga llegue hasta un día después de su llegada, por lo que el tiempo promedio es de 0.33 días cuando el punto de llegada es el terminal portuario y 2.6 días cuando el depósito temporal es el punto de llegada.

Para el caso del canal rojo, el 3.26\% de las declaraciones anticipadas pasan por este canal de control físico, siendo el tiempo de despachar en puerto de 3.26 días, considerando la llegada de la nave y 5.9 días cuando el despacho ocurre en los depósitos temporales. 


\section{Gráfico Nº4}

Tiempo promedio de despacho anticipado por semana.

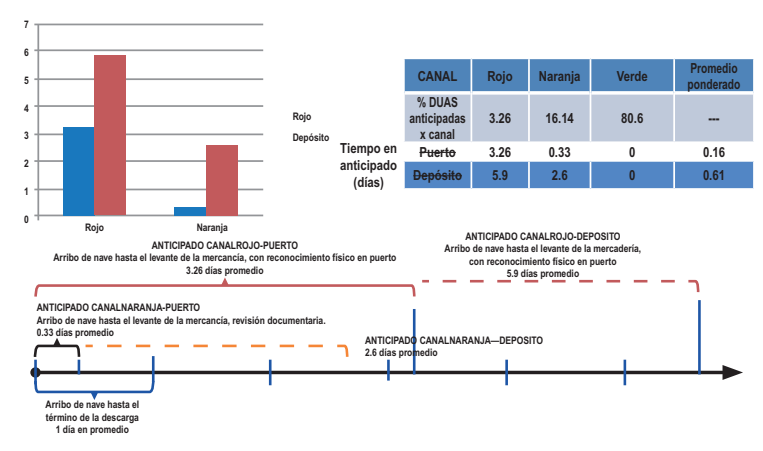

Fuente:SUNAT.

Elaboración: Apoyo Consultoría.

\section{Limitaciones encontradas}

Tratando de darle una explicación a este hecho, se recurrió a información extraída de la SUNAT y de la literatura especializada (BID, 2010), llegando a las siguientes conclusiones:

- Poco conocimiento en los requisitos de este sistema. Algunos agentes de aduanas han manifestado que no usarían este sistema hasta conocerlo mejor y evaluar su aplicación.

- Desconocimiento de los requisitos y facilidades de las garantías globales y específicas. Muchos importadores y agentes de aduanas han manifestado que no han recibido una adecuada instrucción del uso de este mecanismo y prefieren no utilizarlo por el elevado costo que implica la obtención de las garantías financieras.

- Demora en la obtención de permisos especiales para el caso de mercancías restringidas por las diversas autoridades (Ministerio de Transporte y Comunicaciones, Ministerio de Salud, Ministerio de la Producción, etc.). Asimismo, no hay una adecuada difusión, implementación y uso de la Ventanilla Única de Comercio Exterior - VUCE.

\section{Medidas a tomar}

Si bien existe la voluntad de las autoridades aduaneras de minimizar el tiempo de desaduanaje y cumplir en lo posible el levante dentro de las cuarenta y ocho (48) horas, las normas emitidas y las acciones realizadas hasta el momento no han permitido que se logre incrementar el uso de este sistema. Para ello, se debería:

- Propagar mayor conocimiento de cómo trabaja el sistema de garantías.

- Disminuir o eliminar el alto costo de obtener vistos buenos, y el traslado físico para que estos sean pagados en las oficinas de los agentes marítimos, los que se encuentran dispersos por todo Lima.

- Ampliar el horario de atención aduanera, especialmente para el caso de despachos anticipados, pues los plazos para transmitir información son muy cortos.

- Emitir una norma que disponga e incentive el uso del despacho anticipado en puerto, debiendo los agentes marítimos adecuarse y estandarizar sus plazos y trámites.

\section{b. Despacho excepcional (Diferido)}

En el despacho excepcional, las mercancías son solicitadas a régimen de importación una vez arribada la nave. Esta modalidad de despacho es una de las innovaciones que introdujo el actual Decreto Legislativo $\mathrm{N}^{\circ} 1053$ (Ley General de Aduanas, 2008), toda vez que antes de la promulgación de esta norma, todos los despachos debían ser destinados a importación cuando la mercancía se encontraba en territorio peruano.

Ahora bien, la gran diferencia entre el despacho anticipado, el .despacho urgente y el despacho excepcional también radica en el flujo operativo de la carga desde su llegada hasta el levante de la mercancía, tal como se puede apreciar en el gráfico $\mathrm{N}^{\circ} 05$ : 
Figura 5 . Modalidades de despacho aduanero

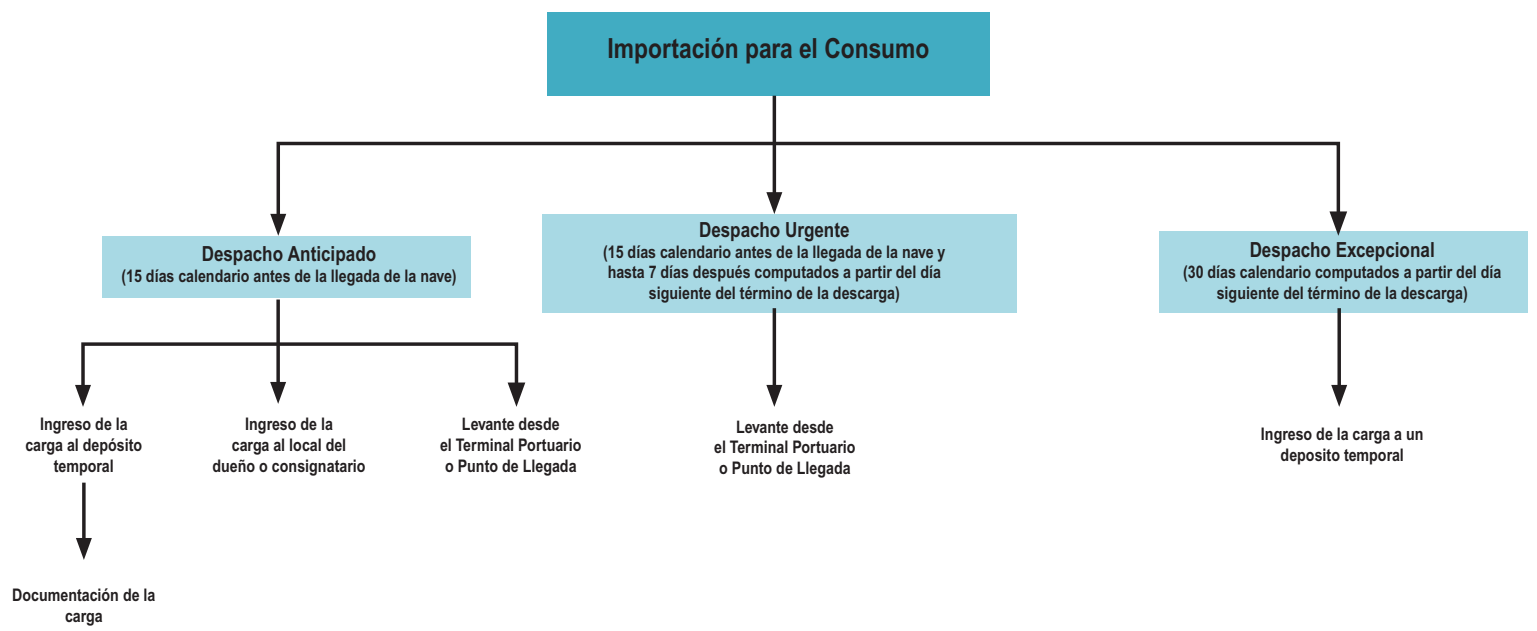

Fuente: Veritra de Analytics

Elaboración: Apoyo Consultoría

Así, en el caso de despacho anticipado, se ha señalado que no era necesario que una mercancía ingrese a un depósito temporal, pues este podía ser retirado desde el punto de llegada designado por el dueño o consignatario, o en su defecto, el designado por el transportista, o incluso ser llevado directamente al almacén del dueño para culminar las acciones de control y operaciones aduaneras propias del levante.

Sin embargo, en el caso del despacho excepcional, si se requiere que la mercancía, una vez arribada y descargada en el puerto, sea trasladada a un depósito temporal, ya sea que este se ubique dentro o fuera del puerto (extraportuario). En estos casos, ya no solo se garantiza que la mercancía sea retirada dentro del plazo de cuarenta y ocho (48) horas, sino que, además, se pueden ir generando costos y sobrecostos por las operaciones que se realicen en este recinto.

Asimismo, la naturaleza de la mercancía o su destinación puede ser un indicador para determinar si debe ser trasladado a un depósito temporal. En efecto, el artículo 114 de la Ley General de Aduanas establece los siguientes casos:

- Cuando se trate de carga peligrosa y este no puede permanecer en el puerto.

- Cuando se destine al régimen de depósito aduanero ${ }^{4}$.

- Cuando se destine con posterioridad a la llegada de la nave (despacho excepcional).

- Otros que se establezca mediante Decreto Supremo.

\section{DISCUSIÓN}

En la actualidad, existen diversos factores que inciden en la dinámica del comercio exterior peruano, entre ellos está la diversidad de las mercancías negociadas, la capacidad de los medios de transporte, el rendimiento en las operaciones de carga y descarga y la tecnología en la transmisión de información electrónica, entre otros.

De acuerdo a la información obtenida de otros estudios, se ha determinado que el costo por el servicio de depósito temporal que brindan los propios terminales portuarios es menor que los cobrados por los depósitos temporales extraportuarios. Sin embargo, lo cierto es que a pesar de la diferencia existente entre los costos del depósito temporal portuario y el extraportuario, la mayor cuota de mercado por este servicio la tienen los depósitos temporales extraportuarios. La utilización del despacho anticipado beneficia a los importadores, en cuanto existe una considerable reducción de determinados costos; sin embargo, existen algunos factores que impiden y hacen poco ágil y, consecuentemente, poco atractiva su utilización. La gran diferencia entre el despacho anticipado, el despacho urgente y el despacho excepcional radica en el flujo operativo de la carga desde su llegada hasta el levante de la mercancía.

\section{CONCLUSIONES}

1. Nuestro país se encuentra en un proceso de crecimiento y dinamización de su comercio exterior, razón por la cual debe 
llevarse a cabo la adecuación de determinadas actividades operativas y aduaneras a esta nueva realidad. 2 . En la actualidad, existen diversos factores que inciden en la dinámica del comercio exterior peruano, estos factores generan la necesidad de aceleración en las operaciones portuarias y la simplificación de las actividades aduaneras. 3. De acuerdo a la información obtenida en otros estudios, se ha determinado que el costo por el servicio de depósito temporal que brindan los terminales portuarios es menor que los cobrados por los depósitos temporales extraportuarios; sin embargo, lo cierto es que, a pesar de la diferencia existente entre los costos del depósito temporal portuario y el extraportuario, la mayor cuota de mercado por este servicio la tienen los depósitos temporales extraportuarios. 4. Los usuarios desconocen las ventajas económicas que podrían obtener al gestionar sus despachos a través del depósito temporal ubicado en el terminal portuario, ya que no asimilan o desconocen el modelo del operador portuario actual, quien garantiza la capacidad suficiente y logística para que gestionen sus despachos directamente desde las instalaciones portuarias, sin necesidad de recurrir a un recinto especial diferente al puerto. 5 . La utilización del despacho anticipado beneficia a los importadores, en cuanto existe una considerable reducción de tiempo y determinados costos, sin embargo, existen algunos factores que impiden y hacen poco ágil y, consecuentemente, poco atractiva su utilización. 6. Uno de los motivos por los cuales los despachadores de aduanas prefieren seguir utilizando la modalidad de despacho excepcional, en lugar del despacho anticipado, es la costumbre en el uso de esta modalidad, ya que la mayoría de las declaraciones de aduanas numeradas en un año son en su mayoría numeradas luego del arribo de la nave.

\section{RECOMENDACIONES}

1. La necesidad de acelerar la dinámica de las operaciones portuarias, así como la simplificación de las actividades aduaneras es de vital importancia, ya que al cubrir estos requerimien- tos se generaría como resultado la reducción de determinados costos y sobrecostos en la cadena logística del comercio internacional. 2. Existe la necesidad de que la Aduana perfeccione su normativa y procedimientos a efectos de que los distintos operadores de comercio exterior puedan ofrecer y prestar servicios más complejos de una forma más ágil. 3. Es conveniente difundir las ventajas de gestionar los despachos de mercancías a través del depósito temporal ubicado en el recinto portuario.

\section{REFERENCIAS BIBLIOGRÁFICAS}

BANCO INTERAMERICANO DE DESARROLLO - BID. 2010. Barbero, J. A. Notas Técnicas No IDBTN-103: La logística de cargas en América Latina y El Caribe: una agenda para mejorar su desempeño. Págs. 27-34, 41-42, 45-51.

Código de Procedimientos Aduaneros. 1921. Ley 4380.

Fusión por absorción de la Superintendencia Nacional de Administración Tributaria SUNAT con la Superintendencia Nacional de Aduanas - ADUANAS. 2002. Decreto Supremo $N^{\circ}$ 061-2002-PCM.

Huamán Sialer, M. A. 2011. LEX Revista de la Facultad de Derecho y Ciencia Política - UAP. Vol. 9. Núm. 8-Lima. Págs. 360-361.

Ley de Creación de la Superintendencia Nacional de Aduanas. 1988. Ley № 24829.

Ley Orgánica de la Superintendencia Nacional de Aduanas. 1992. Decreto Ley No 26020.

Ley General de Aduanas. 2008. Decreto Legislativo No 1053. Art. 15 - 46, 47 - 97, 114 y 167.

Ley de Facilitación del Comercio Exterior. 2004. Ley $\mathrm{N}^{\circ} 28977$.

Ministerio de Comercio Exterior y Turismo - MINCETUR. 2005. Sgut, M. Estudio de Costos y Sobrecostos Portuarios en el Puerto del Callao. Pags. 26-38.

Procedimiento General de Exportación Definitiva - INTA.PG.02., 2009.

Procedimiento General de Importación Definitiva - INTA.PG.01., 2009.

Reglamento de la Ley General de Aduanas. 2009. Decreto Supremo No 010.2009-EF. Art. 6 
